\title{
Lock-in amplifiers for AMR sensors
}

\author{
Marina Díaz-Michelena ，Pedro Cobos ,Claudio Aroca
}

\begin{abstract}
A B S T R A C T
Anisotropic magnetoresistive (AMR) magnetic sensors are often chosen as the magnetic transducer for magnetic field sensing in applications with low to moderate magnetic field resolution because of the relative low mass of the sensor and their ease of use. They measure magnetic fields in the order of the Earth magnetic field (with typical sensitivities of $1 \% / \mathrm{G}$ or $10^{-2} \% / \mu \mathrm{T}$ ), have typical minimum detectable fields in order of $\mathrm{nT}$ and even $0.1 \mathrm{nT}$ but they are seriously limited by the thermal drifts due to the variation of the resistivity with temperature $\left(\sim 2.5 \%\right.$ o $\left.{ }^{\circ} \mathrm{C}\right)$ and the variation of the magnetoresistive effect with temperature (which affects both the sensitivity of the sensors: $\sim 2.7 \% /{ }^{\circ} \mathrm{C}$, and the offset: $\pm 0.5 \% /{ }^{\circ} \mathrm{C}$ ). Therefore, for lower magnetic fields, fluxgate vector sensors are generally preferred.

In the present work these limitations of AMR sensors are outlined and studied. Three methods based on lock-in amplifiers are proposed as low noise techniques. Their performance has been simulated, experimentally tested and comparatively discussed. The developed model has been also used to derive a technique for temperature compensation of AMR response. The final goal to implement these techniques in a space qualified applied specific integrated circuit (ASIC) for Mars in situ exploration with compact miniaturized magnetometers.
\end{abstract}

\section{Introduction}

Anisotropic magnetoresistance (AMR) is the change of electrical resistance due to the variation of the magnetization vector projection on the electrical current direction. It is an effect due to the spin-orbit coupling (Fig. 1). When an electrical current passes through a material, the transport electrons experience a scattering. This scattering is a function of the distribution of the electronic clouds in the material and this distribution is related to the magnetization.

The magnetoresistive effect can be observed in all the materials. However, its intensity is different in the distinct materials. The alloy of niquel and iron named permalloy presents a high magnetoresistive effect in the order of $2-3 \%$, which makes it suitable for magnetic sensors based on this effect. The permalloy has an internal anisotropy, which tends to align the magnetic dipoles in a certain direction called easy axis of magnetization or simply easy axis. In the presence of an external field, the permalloy acquires magnetization against this anisotropy. Let $U$ be the magnetic energy density, $K$ the anisotropy constant, $H$ the magnetic field, $M_{S}$ the saturation magnetization, $\varphi$ the angle between the magnetization vector and the easy axis. The expression for the energy density of every permalloy resistor exposed to a magnetic field $\mathrm{H}$ has two terms in opposition: one related to the anisotropy energy and another to the magnetostatic energy associated to the effect of the field:

$U=K \cos ^{2}(\varphi)-M_{S} H \cos (\varphi)$

If one derives this expression and equals the derivative to zero, to find the equilibrium orientation of the magnetization vector in the presence of an applied field $H$, it is found that the equilibrium angles for the magnetization vector respect to the easy axis are:

$\varphi=0, \arccos \left(\frac{M_{S} H}{2 K}\right)$.

The changes in the electrical resistance are an indirect measurement of the magnetic field because the resistance in the permalloy cores can be expressed as a function of $\varphi$ :

$R(\varphi)=R_{0}+R_{\Delta} \cos ^{2}(\varphi)$

where $R$ is the measured resistance, $R_{0}$ is the contribution of the electrical resistance in the absence of a magnetic field and $R_{\Delta}$ is the magnetoresistive contribution. This contribution can be explained as the difference in the resistivity of the material depending on the relative direction between magnetization and current, being 


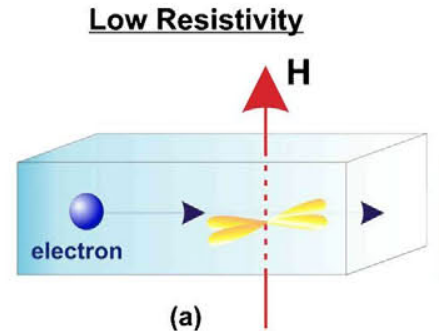

High Resistivity

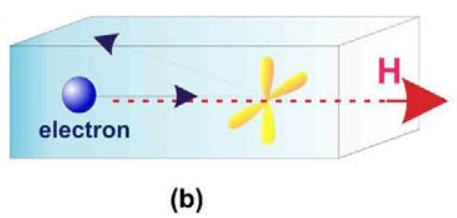

Fig. 1. AMR Spin-orbit coupling.

$\rho_{\| M}$ the resistivity of the material when the current flows in the direction of the magnetization and $\rho \perp_{M}$ the resistivity of the material when the current flows perpendicularly to the magnetization vector (Fig. 2a).

Let the direction between contacts (Fig. 2a) be that of the easy axis (shape anisotropy) of the permalloy core and the perpendicular direction, the sensing direction. Since AMR is an even effect it cannot discern between fields of different signs. To transform the measurement in an odd effect, the permalloy straps usually have a Barber pole biasing, which consists in an array of copper microstraps inserted in the permalloy at $45^{\circ}$ (i.e. $\pi / 4 \mathrm{rad}$ ) respect to the line between the electrical contacts forcing the electrical current to pass through the film at $a-45^{\circ}$ (Fig. 2a).

The sensors are provided with a coil named set-reset strap to magnetize the permalloy core in such a way that the magnetic spins are firstly aligned in the easy axis direction to linearize the response curve vs. the magnetic field and to avoid Barkhaussen noise due to the energy required to move the magnetic domains walls. This can be performed with a short current pulse of intensity high enough so as to saturate the permalloy core in the easy axis direction. The flipping can be performed in both directions alternatively (set-reset operation) to reduce the offset and the hysteresis.

Following the scheme of Fig. 2a, the resistance of a single permalloy core in the presence of an external magnetic field $H$ would have complementary projections after a set pulse or a reset pulse, applied in the opposite direction:

$R_{\text {reset }}=R_{\Delta} \quad \cos ^{2}\left[\operatorname{arcos}\left(\frac{M_{S} H}{2 K}\right)-\frac{\pi}{4}\right] \quad$ and

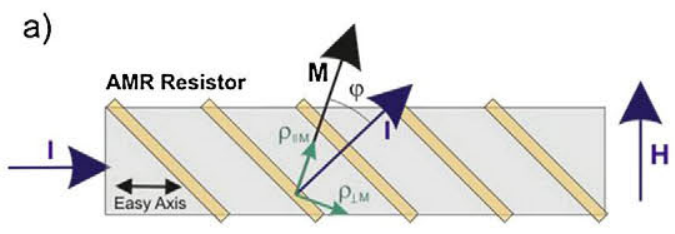

b)

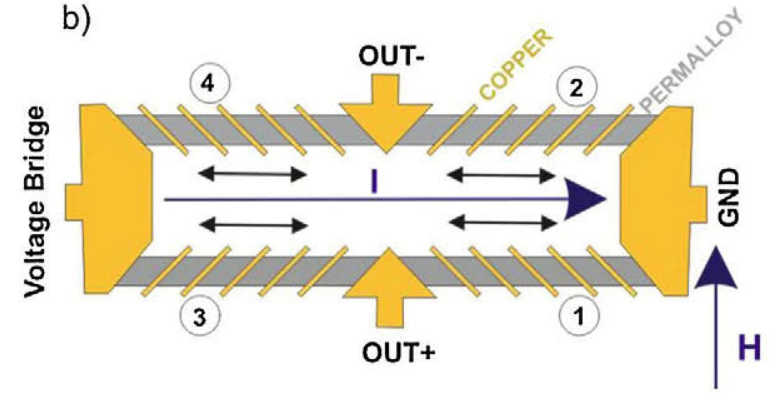

Fig. 2. (a) AMR device indicating current, magnetization directions and the resistivity parallel and perpendicular to the magnetization, (b) Wheatstone bridge of AMRs: Resistors 1 and 3 are magnetically biased in one direction and 2 and 4 in the perpendicular one.

$$
R_{\text {set }}=R_{\Delta} \sin ^{2}\left[\operatorname{arcos}\left(\frac{M_{S} H}{2 K}\right)-\frac{\pi}{4}\right]
$$

The normalized resistance as a function of the magnetic field after a reset pulse is plotted in Fig. 3 (a: simulation, b: experimental curve using a commercial HMC1002 sensor by Honeywell). Note that even though the equations are given as a function of the magnetic field, in the graphs it is represented the magnetic induction since most users usually give sensitivities as a function of Oersteds or Teslas. Also, in our experimental set ups $B$ is proportional to $H$ being the proportionality constant the vaccum permeability $\mu_{0}$.

The most common way to measure the resistance is by means of a Wheatstone bridge so most of the AMR commercial devices have a Wheatstone bridge with four magnetoresistors (Fig. 2b).

The four permalloy cores in the Wheatstone bridge are trimmed and prepared for being magnetically biased in couples (Fig. 2b).

In the presence of a magnetic field the cores experiment a change in their magnetization state and the differential voltage of the bridge is:

$V_{\text {set } / \text { reset }}=V_{+}-V_{-}=\left[\frac{R_{1}}{R_{1}+R_{3}}+\frac{R_{2}}{R_{2}+R_{4}}\right] V_{\text {bridge }}$

At room temperature this differential voltage for permalloy cores of several tens of nanometers is in the order of $2 \%$ of the bridge voltage, which makes them suitable candidates for measuring fields of low to moderate intensity [1,2]. The typical resolutions reported are in the order of nT [3] and some works have achieved tenths of nT resolutions [4].

To achieve the best resolution the sensors are often run in a continuous reset and set mode. This is performed by a set of current pulses of duration in the order of $\mu$ s and an intensity enough to saturate the cores in their easy axis and both directions alternatively. In set-reset operation the noise of AMR sensors is reduced considerably [5].

In this operation mode the output voltage of the Wheatstone bridge is:

$V_{\text {OUT }}=\frac{\left\langle V_{\text {set }}\right\rangle-\left\langle V_{\text {reset }}\right\rangle}{2}$

where $\left\langle V_{\text {set }}\right\rangle$ and $\left\langle V_{\text {reset }}\right\rangle$ are the averaged measurements after the set and reset pulse respectively. This operation increases slightly the difficulty of use of AMRs and reduces the bandwidth since the acquisition frequency is limited by the time needed to acquire both sets of samples.

Finally, the commercial devices have also another coil with the double purpose of zeroing the bridge, auto-calibration and feedback. In this work a feedback circuitry is not considered in principle on the first hand due to the good linearity of the sensors: better than $0.1 \%$ at full scale for fields lower than $100 \mu \mathrm{T}$ and on the other hand because it does not simplify the circuitry but translates the strong requirements in terms of bits needed to the current source. Other groups have performed very interesting works in this line [6-8].

So far it has been described a typical commercial AMR sensor at a certain temperature but AMR sensors are highly dependent on the temperature. Many properties of the AMR cores are a function of temperature like the saturation magnetization, the resistance, the anisotropy, etc. The AMR output therefore, is influenced by these parameters temperature varying. This is illustrated in Fig. 4 with $10 \%$ variations of some of the parameters. This question is especially important if the application implies wide temperature ranges like the daily temperature variations on the surface of Mars ( $190 \mathrm{C}$ to $20 \mathrm{C}$ ), and these sensors are competitive sensors for magnetic measurements on the surface of Mars because of their low mass and power consumption.

Fig. 4 a shows the change in the output signal of an AMR (after a reset) for a $10 \%$ change of saturation magnetization (i.e. Ms $=1-$ red and $0.9 \mathrm{~T}-$ green), assuming that the permalloy cores only have 

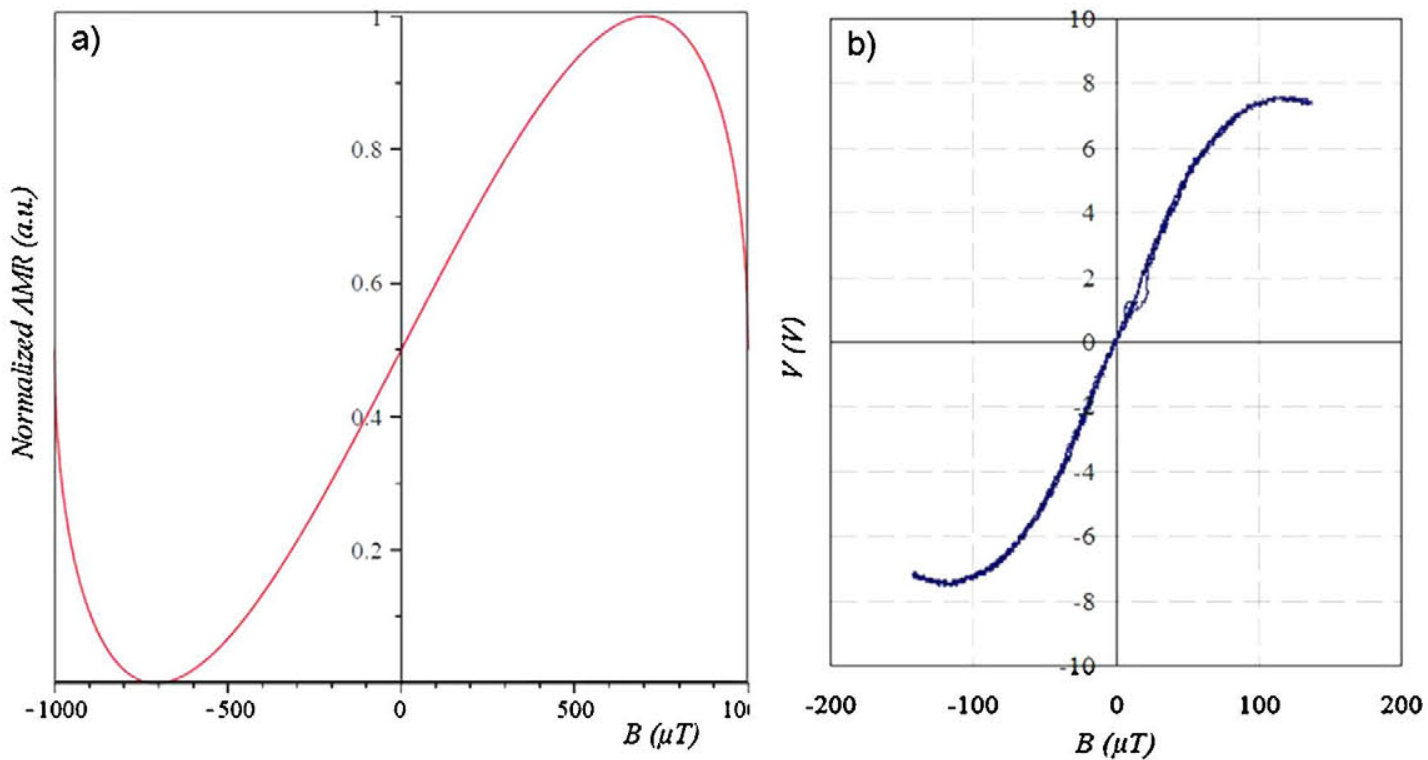

Fig. 3. (a) Theoretical normalized reset versus magnetic field, (b) experimental measurement of the reset with a $\mathrm{HMC} 1002$ sensor (Saturating field: $\pm 100 \mu \mathrm{T}$ ).

shape anisotropy and then the anisotropy constant can be taken as:

$k=\frac{N M_{s}^{2}}{\mu_{0}}$

where $N$ is the shape factor (estimated in $N=5 \times 10^{-4}$, in view of the fact that the saturating field: $H_{s}$, is in the order of $1000 \mu \mathrm{T}$, that $H_{s}$ is the sum of anisotropy and demagnetizing fields of the same order of magnitude, and assuming that the induction of the demagnetization is $1 \mathrm{~T})$ and $\mu_{0}$ is the vacuum permeability $\left(\mu_{0}=4 \pi 10^{-7} \mathrm{H} / \mathrm{m}\right)$. Taking this into account reset and set measurements results in:

$R_{\text {reset }}=R_{\Delta} \cos ^{2}\left[\operatorname{arcos}\left(\frac{\mu_{0} H}{2 N M_{S}}\right)-\frac{\pi}{4}\right]$

and

$R_{\text {set }}=R_{\Delta} \cos ^{2}\left[\operatorname{arcos}\left(\frac{\mu_{0} H}{2 N M_{S}}\right)+\frac{\pi}{4}\right]$

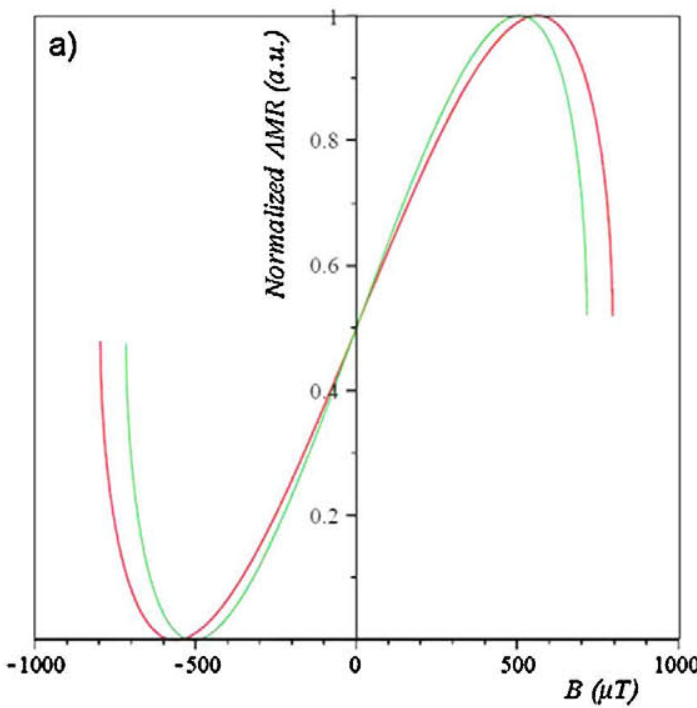

$=R_{\Delta} \sin ^{2}\left[\operatorname{arcos}\left(\frac{\mu_{0} H}{2 N M_{S}}\right)-\frac{\pi}{4}\right]$

Fig. $4 \mathrm{~b}$ shows the change in the output signal of an AMR (after a reset) measurement for a $10 \%$ of the normalized resistance variation $\left(R_{\Delta}=1 \Omega\right.$-red and $R_{\Delta}=0.9 \Omega$-green $)$.

The AMR sensors have typical sensitivities of $1 \% / G / V_{\text {bridge }}$ or

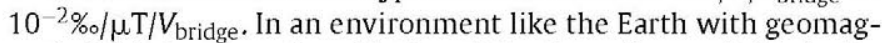
netic fields in the order of $1 \mathrm{G}$ or $100 \mu \mathrm{T}$, to discern fields in the order of $10 \mu \mathrm{G}$ or $1 \mathrm{nT}$ implies to detect 5 parts in $10^{8}$ if the voltage bridge is $5 \mathrm{~V}$. But the signal due to the magnetoresistive effect will be perturbed by the resistance of the magnetoresistive elements, which will suffer changes not only because of the variation of the magnetoresistance with temperature (which affects both the sensitivity of the sensors: $\sim 2.7 \% \circ{ }^{\circ} \mathrm{C}$, and the offset: $\pm 0.5 \% \circ /{ }^{\circ} \mathrm{C}$ ) but also because of the resistivity change with temperature $\left(\sim 2.5 \% /{ }^{\circ} \mathrm{C}\right)$. Theoretically for perfectly trimmed resistors, the offset of the sensors (output of the sensor in the absence of magnetic field) should be independent of the temperature but unfortunately the trimming

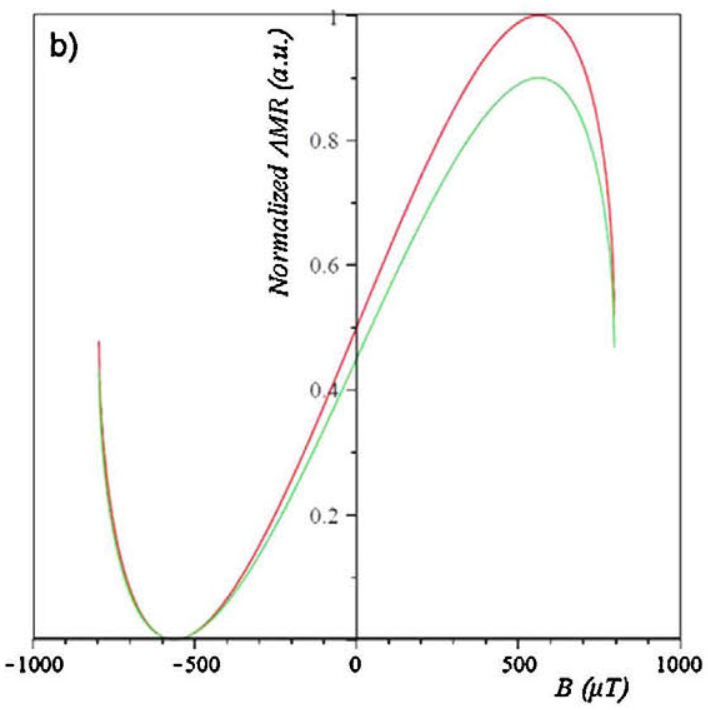

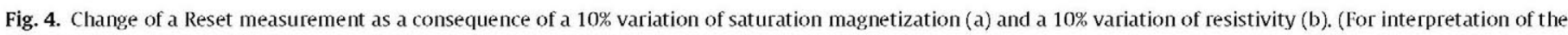
references to color in this figure legend, the reader is referred to the web version of this article.) 


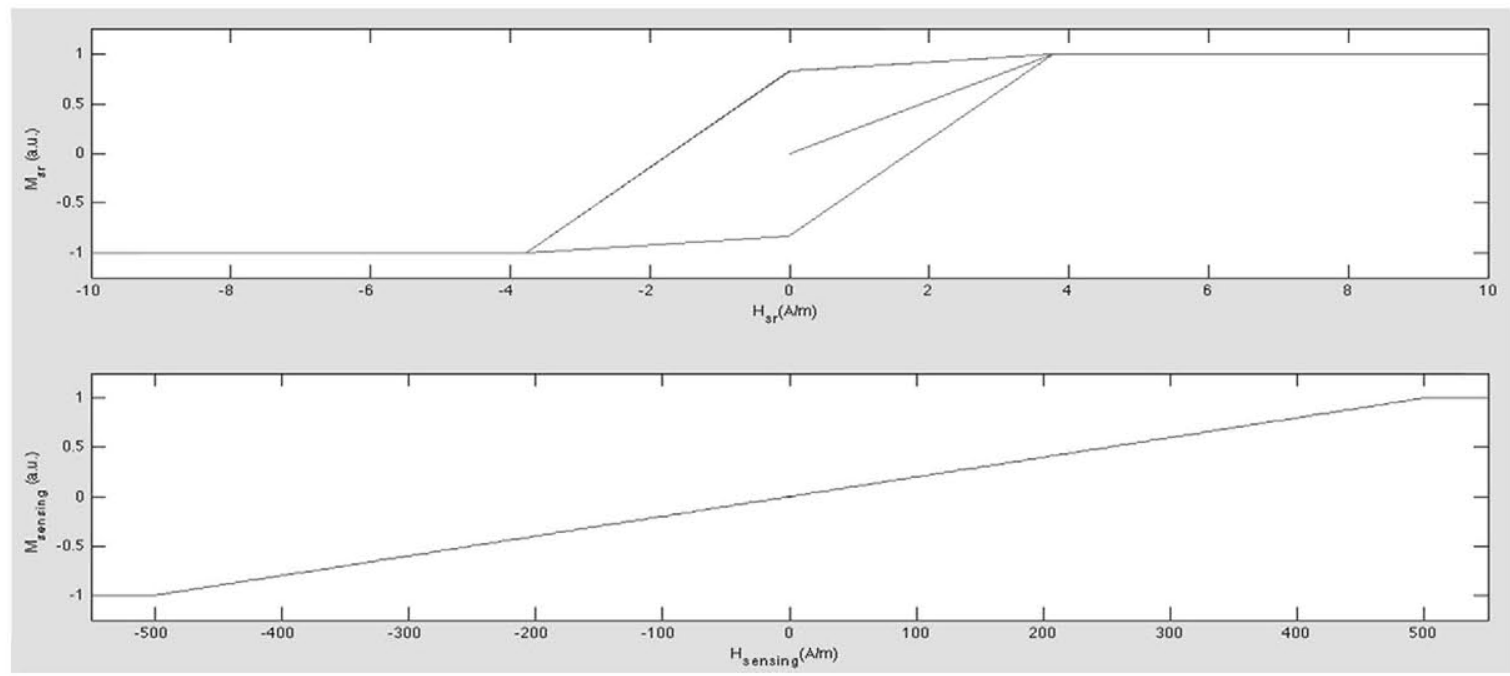

Fig. 5. Hysteresis loops of the AMR strap when magnetized in the easy axis direction (upper graph) and in the sensing direction (lower graph).

is not perfect as it can be seen in the zero field response, and can have errors in the order of 6\% [9]. The unbalance is proportional to the resistance value having a stronger influence for small values of resistors. This double effect implies a limitation of the sensors sensitivity, which is enhanced for applications with operations in high temperature swing $[10,11]$.

Graphs of Fig. 4 show that the variation of the measurements with temperature is very complicated and this limits the performance of AMR in applications with a wide temperature swing. Besides the question with temperature is tricky in the sense that except for the situations when the sensor is run in thermal equilibrium, a measurement of temperature outside the sensor is not representative of the temperature of the Wheatstone bridge and thus measurements of the same external field during heating or cooling of the sensors may have different outputs. In previous works it has been used an external temperature sensor for thermal compensation [1] but there was a non-negligible error in the measurement of the bridge temperature. In this work we propose to measure the temperature by means of the change of resistance of the whole Wheatstone bridge.

The other critical factor that needs to be taken into account is the noise.

As every magnetic sensor, AMR devices present a $1 / f$ noise $[12,13]$ and thus, for bandwidths of tens of $\mathrm{Hz}$ the signal to noise ratio (SNR) increases with frequency measurements. In this sense, any kind of lock-in amplifier or sigma-delta [8] circuitry can help to reduce the noise of both open and closed loop circuits as will be discussed below.

In this work we report on some lock-in based set ups for improving AMR sensors response focusing on the increase of the SNR and the temperature immunity of the measurements. The final goal is to squeeze AMR sensors performance, which have a potential for miniaturization higher than that of fluxgates [1,2], and try to widen the field of AMR sensors in applications of low field detectivity, where fluxgates are preferred [14,15].

\section{Experimental methods and results}

In this work it has been simulated and experimentally tested three different lock-in based set ups with AMR sensors:

1. A lock-in amplifier with the set-reset flipping.

2. Fluxgate like operation of the AMR.

3. A hybrid method combining set-reset flipping and fluxgate operation.

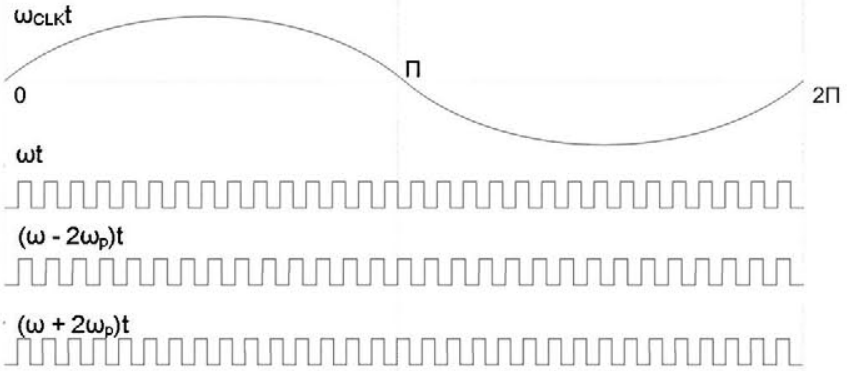

Fig. 6. Squared waveforms generated by a clock.

The ultimate goal is to do a comparative study of their performance and to find solutions to the temperature dependency of AMR devices. Along the three next subsections simulations and experimental results will be shown. The model used to simulate the AMR devices response is compared successfully with experimental results. Based on this validation, the model has been used to derive a method to compensate AMR response with temperature. Further studies in thermal and magnetically controlled chambers will have to contrast experimentally the predictions of the simulations.

The simulations have been performed with Matlab and MAPPLE. The AMR device has been modelled as a soft magnetic material with shape anisotropy. The hysteresis loops of the core in the easy axis and in the sensing direction can be seen in Fig. 5. (Notice change of scale in the horizontal axis).

The magnetoresistive effect has been modeled as indicated in the previous section (Eqs. (1)-(7)). To simplify, in the model we refer to $R_{\Delta}$ as the resistance, because it is the term in Eq. (2), which corresponds to the magnetoresistive effect. The outputs of the lock in amplifiers will include the calculation of the corresponding integrals of the signal (Eq. (7)) in the corresponding time frames.

Method described in Section 2.3 is based on two simultaneous and perpendicular excitations of the AMR device: a set-reset squared carrier signal (in the easy axis) and a biasing sine modulating signal (in the sensing direction). To avoid synchronism problems in the experimental set up a single clock has been used to generate all the needed frequencies. The exciting signals are calculated in such a way that their frequencies are integers and linearly dependent of the other (Fig. 6). This is achieved by means of the solution of a Diophantine equation system. Many combinations 


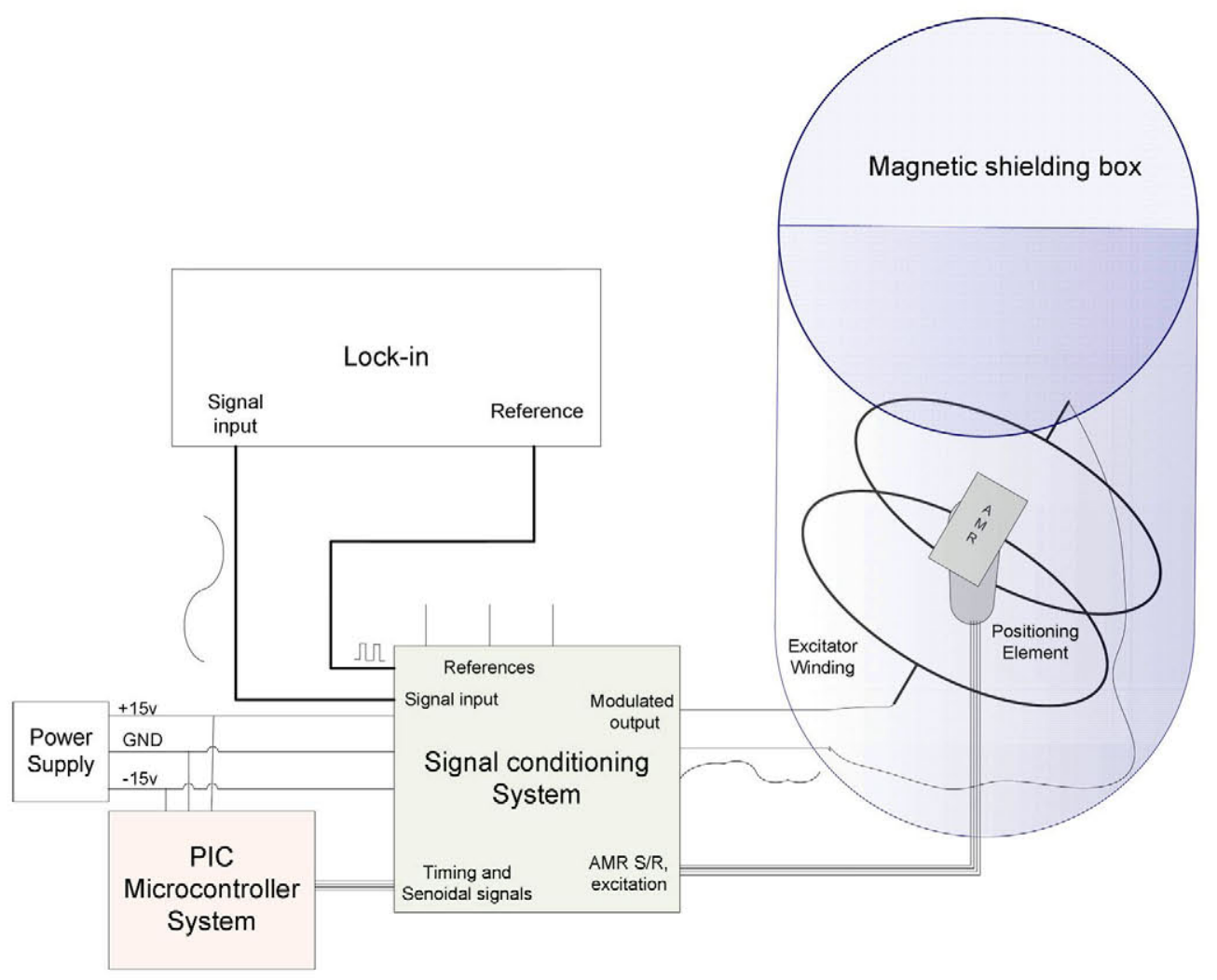

Fig. 7. Set up for the measurements. The magnetic shielding chamber was used in minimal signal detection.

have been tested, trying to generate as many as possible sequences inside a sine period, the problem being to find integer numbers, for instance $192,32,24,48$ that permit the correct generation of the signals. In this case, for instance, 192 clock cycles are equivalent to a sine period, 32 to generate changes in $\omega$, and so on.

The set up built up specifically for the measurements (Fig. 7) consists of three electronic printed circuit boards (PCB):

\section{a. AMR PCB including a commercial AMR biaxial sensor HMC1002} by Honeywell [18].

b. Signal Conditioning System PCB.

c. Microcontroller System PCB,

and one or two lock-in amplifiers for demodulation or double demodulation.

Apart from the AMR sensor, the AMR PCB contains a frontend analog circuitry. The design of this PCB, highly miniaturized, is intended to avoid interferences in the sensor. The wires that link this PCB with other elements have been carefully twisted and grounded. In principle this PCB has been placed in a magnetic shielding chamber. For measurements out of the chamber, the Earth magnetic field has been properly compensated.

The Microcontroller System PCB consists of a PIC16F887 microcontroller of 8 bits by Microchip, running at $8 \mathrm{MHz}$, programmed and debugged using the In System Programming method. As it has been introduced, to avoid synchronism problems, the developed application obtains all the required timing signals (diophantine solutions) from a single clock using a 12 bit sampling of the clock sine wave (Fig. 6). The program is susceptible to be modified with a laptop.

The sine values samples (12 bits) are stored at the microcontroller internal memory, and they are read to produce the digital output for the Digital-to-Analog (D/A) converter. In parallel, the digital signals are generated.
Finally the signal conditioning system PCB adapts both the AMR and the reference signals according to the lock-in amplifier requirements in an open loop scheme. Thus, it contains a set of analog circuitry for signals adjust. It requires bipolar alimentation $( \pm 15 \mathrm{~V})$ obtained from an external source and generates all the required voltages for every element. Also it contains a DAC (AD667) with 12 bits and bipolar output $( \pm 5 \mathrm{~V})$, to provide the analog sine signal that is used to generate the modulated output via a power amplifier, capable to provide up to $3 \mathrm{~A}$ to the reels.

Fig. 8 shows the detailed AMR connections.

\subsection{Set-reset lock-in}

This method consists in an in-phase detection at the set-reset excitation frequency. This method, widely used with this technology, can improve the spectral noise of a normal open loop up to one order of magnitude [16].

Datasheets of commercial AMR sensors specify that due to the small section of the set-reset straps the current (saturating) pulses should not be longer than $2 \mu$ s and the duty cycle should not be higher than $0.1 \%$ [18].

The experimental set up for this method has been adapted to these frequency limitations specified, which limits the frequency to $250 \mathrm{~Hz}$, a period of $4 \mathrm{~ms}$ for set-reset operation, and double frequency for only set or reset operation. The conservative value of the frequency used is $116 \mathrm{~Hz}$.

Instead of a DC field, the applied external field was a squared $2.6 \mathrm{nT}$ peak-to-peak signal of $16 \mathrm{mHz}$. Fig. 9 shows the Fourier transform of the signal measured by an oscilloscope. The scale of the lock-in amplifier is $50 \mu \mathrm{V}$. It is clear that the sensor responds perfectly for amplitudes in the order of nT and it seems to have at least one order of magnitude of margin. In this case, the minimum detectable field has not been measured diminishing gradually the 


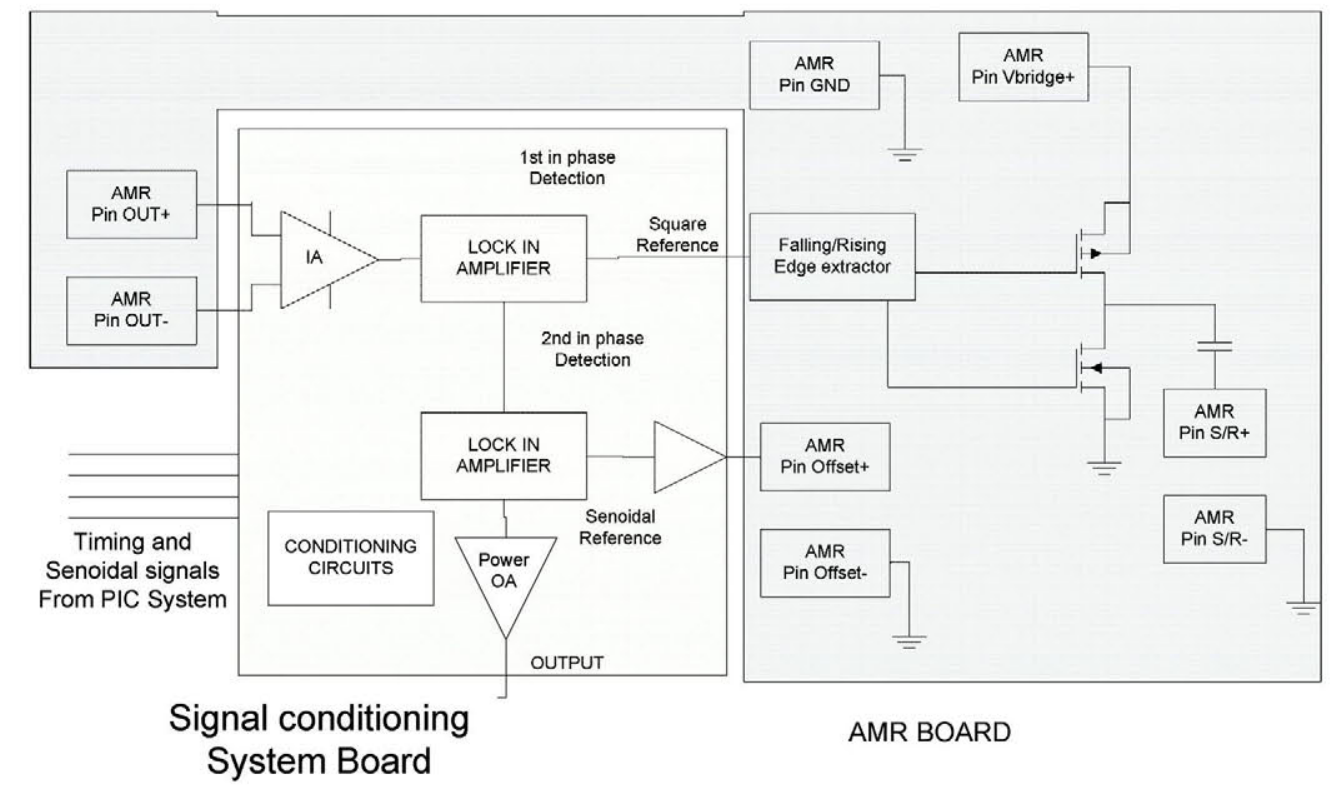

Fig. 8. Excitation AMR circuits.

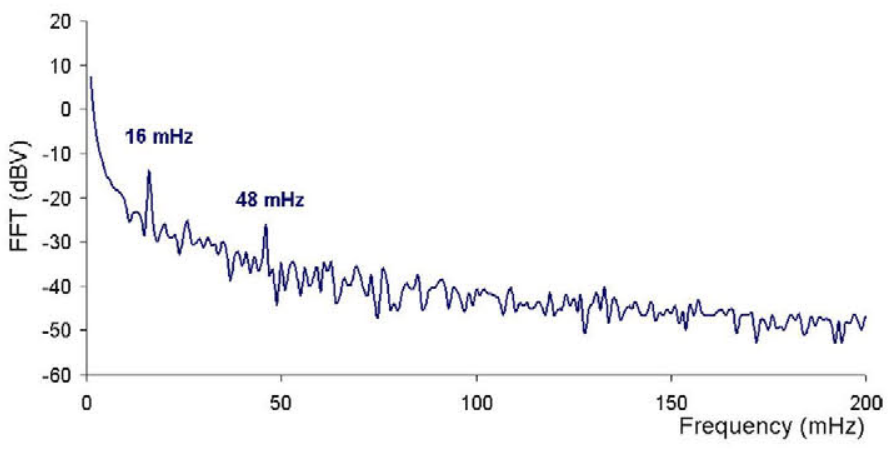

Fig. 9. Fourier transform of the signal for a $2.6 \mathrm{nT}$ amplitude external field of $16 \mathrm{mHz}$. Note that $0 \mathrm{dBv}$ corresponds to $10 \mathrm{nT} / \sqrt{ } \mathrm{Hz}$.

field because we had difficulties to generate lower fields in the laboratory with the available instrumentation.

Despite the good performances with the magnetic field, this set up is very vulnerable to temperature changes.

According to Eq. (5) the output of the AMR device in continuous set-reset operation with a flipping saturating field is a squared signal between the two values specified in Eq. (3).

Obviously, a slight change in the resistance due to a temperature variation will change completely the amplitude of this signal and its projection to the frequency base (lock-in). Thus, even though the set-up is more robust to measure the field at a certain temperature, it is not optimum for the general purpose of measuring the magnetic field in a temperature-changing environment.

\subsection{Fluxgate operation of the AMR}

This method consists in operating the AMR as a fluxgate sensor [17], i.e., saturating alternatively the AMR in its sensing direction by means of an alternating field $\left(H_{0}=700 \mu \mathrm{T}\right.$ and $\left.\omega=2 \pi 10^{3} \mathrm{rad} / \mathrm{s}\right)$. The experimental set-up in this case uses the offset coils to drive the alternative saturation at a frequency $\omega$ and the detection is performed with a lock in amplifier locked at $2 \omega$. When we use set and reset nomenclature we do not refer to the driving to saturation,

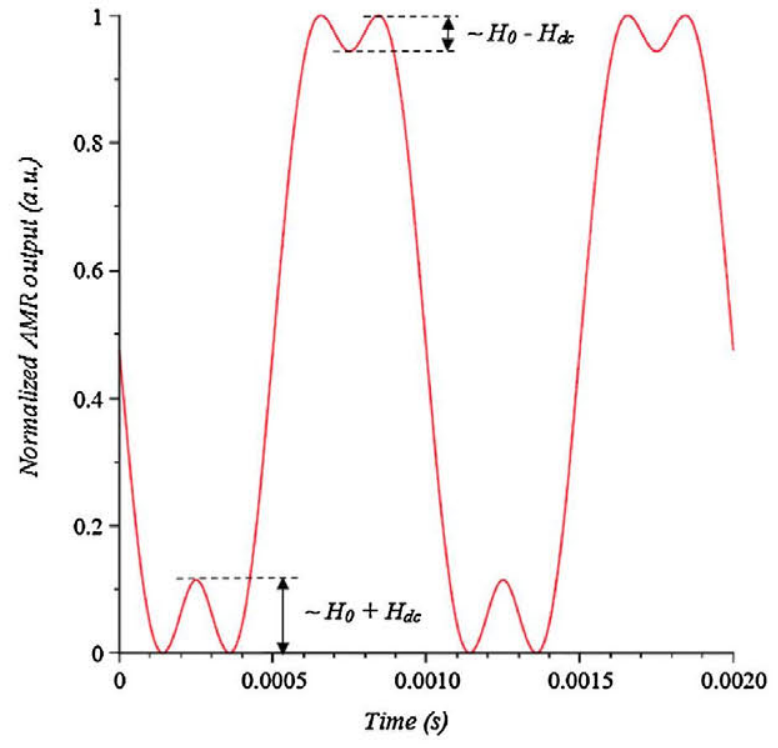

Fig. 10. Normalized AMR output curve for a dc external field superimposed to an alternating saturating field.

which is performed by menas of the offset coils, but to the initial status of magnetization.

The equation of the set signal in this case under the effect of an external field $\left(H_{d c}=20 \mu \mathrm{T}\right)$ is:

$s(t)=R_{\Delta} \quad \cos ^{2}\left\{\arccos \left[\frac{\mu_{0}}{2 N M_{S}}\left[\begin{array}{ll}H_{0} & \sin (\omega t)+H_{d c}\end{array}\right]+\frac{\pi}{4}\right]\right\}$

which results in an asymmetry of the AMR curve (Fig. 10).

This asymmetry produces a second harmonic in the Fourier transform of the signal.

In principle, in this case the sensitivity is higher because the applied field is measured not by an amplitude variation but by a time shift by means of the second harmonic amplitude, whose 


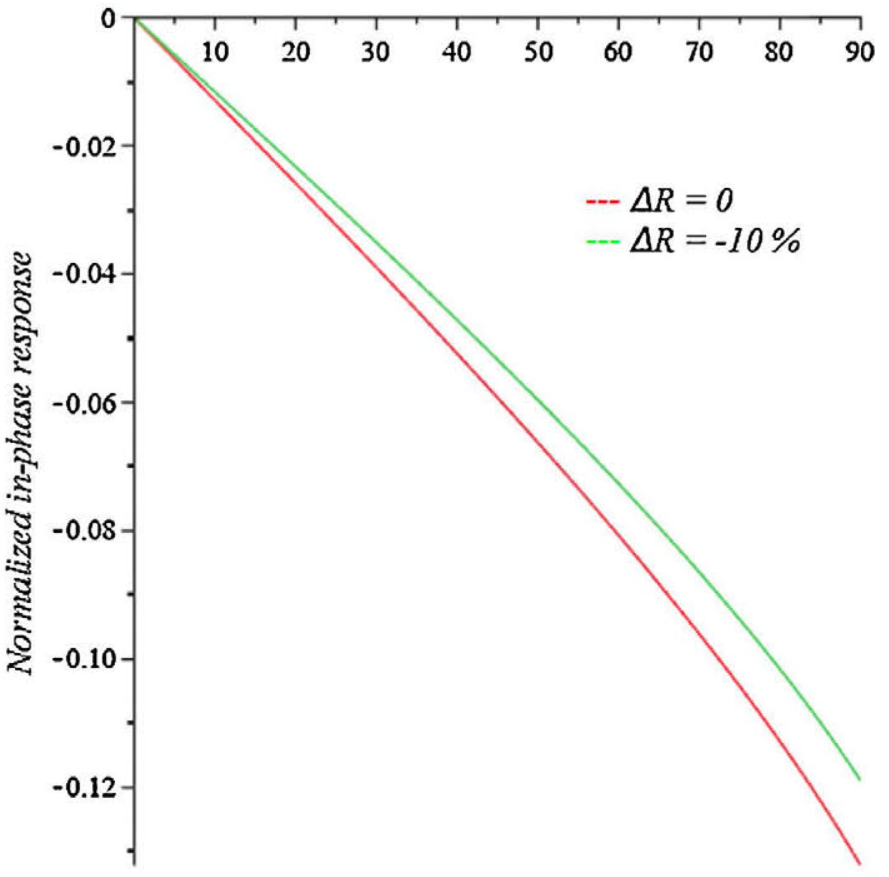

Fig. 11. Normalized in-phase response for a AMR of $R_{\Delta}=1 \Omega$ (red) and $0.9 \Omega$ (green) corresponding to the second harmonic. (For interpretation of the references to color in this figure legend, the reader is referred to the web version of this article.)

components in phase and quadrature are:

$$
\begin{aligned}
s_{\text {in-phase }}(2 \omega) & =\frac{\omega}{\pi} \int_{0}^{2 \pi / \omega} s(t) \sin (2 \omega t) \mathrm{d} t \text { and } s_{\text {in-quadrature }}(2 \omega) \\
& =\frac{\omega}{\pi} \int_{0}^{2 \pi / \omega} s(t) \cos (2 \omega t) \mathrm{d} t
\end{aligned}
$$

In contrast to the previous method (set-reset lock-in), the setreset is not used and the magnetization is not restored in every measurement. This affects negatively the sensitivity, which suffers degradation along the time.

The purpose of this work is not to present new experimental data with this set up since other authors have been reported experimental results with similar set ups [17]. The goal is to use our model to study the temperature dependence and to design a method for its compensation.

Following the previous example, assume a $10 \%$ change in resistance due to a temperature swing. Let $s(t)$ be the signal at the temperature $T_{1}$ and thus, with a $R_{\Delta}$ of $1 \Omega$. The second harmonic is given by Eq. (9). Now let $s^{\prime}(t)$ be the signal at the temperature $T_{2}$ and thus, with a $R_{\Delta}$ of $0.9 \Omega$. Its second harmonic (in phase signal) is given by:

$s_{\text {in-phase }}^{\prime}(2 \omega)=\frac{\omega}{\pi} \int_{0}^{2 \pi / \omega} s^{\prime}(t) \sin (2 \omega t) d t$

The integrated signals as a function of the external field are plotted in Fig. 11, where the red curve corresponds with the reading at $T_{1}$ and the green curve correspons to that at $T_{2}$.

It can be seen that a $10 \%$ change in resistance leads approximately to a $10 \%$ change in sensitivity with field of the AMR measuring the second harmonic.

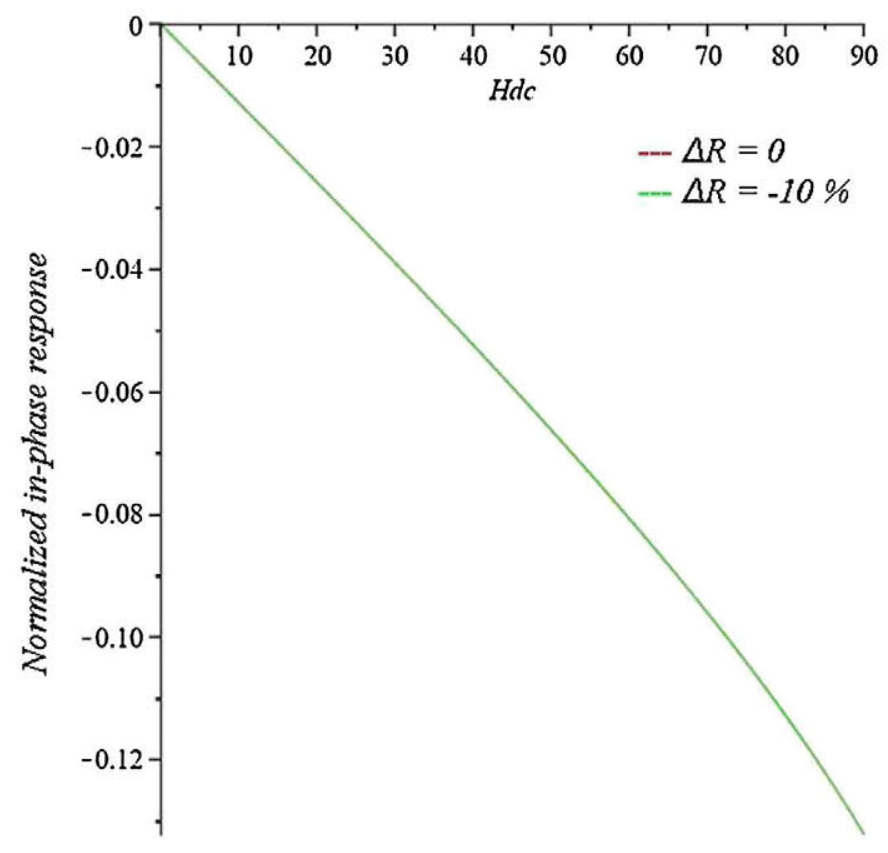

Fig. 12. Normalized in-phase response for a AMR of $R_{\triangle}=1 \Omega$ (red) and $0.9 \Omega$ (green) corresponding to the second armonic of the signal with $R_{\Delta}=1 \Omega$ and the second harmonic corrected by the first harmonic ratio of the signal with $R_{\triangle}=0.9 \Omega$. The two lines are completely overlapped. (For interpretation of the references to color in this figure legend, the reader is referred to the web version of this article.)

A detailed study of the different harmonics of the signal and their behavior with temperature lead to the fact that the first harmonic is insensitive to the field but sensitive to the resistance changes, while the second harmonic is sensitive to both magnitudes. Based on this we propose to normalize the second harmonic with the first harmonic to have a response completely immune to temperature. The normalization factor is the fraction between the first harmonics at $T_{2}$, when $R_{\Delta}=0.9 \Omega$ the and $T_{1}$, when $R_{\Delta}=1 \Omega$ :

$\frac{s_{\text {in-phase }}^{\prime}(\omega)}{s_{\text {in-phase }}(\omega)}=\frac{\int_{0}^{2 \pi / \omega} s^{\prime}(t) \sin (2 \omega t) \mathrm{d} t}{\int_{0}^{2 \pi / \omega} s(t) \sin (2 \omega t) \mathrm{d} t}$

Fig. 12 shows the simulated responses: the red curve represents the in-phase second harmonic at the temperature $T_{1}$ given by Eq. (9) and the red curve represents the in-phase second harmonic at the temperature $T_{2}$ normalized as follows:

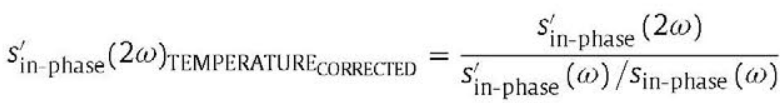

The simulation predicts that there is no observable effect of temperature with this normalization. The method does not provide absolute data because it is relative to the measurement at the former temperature, which is taken as a reference. But it can be used for homogeneous measurements in a wide range of temperatures without the need to measure the temperature but the two first harmonics of the signal. Also, for a finer correction higher order harmonics can be used.

\subsection{Combined set-reset and fluxgate operation}

This last method combines the two previous ones to have the best features of both and solve the problems of the previous methods separately, this is, the temperature compensation in the first method, and the lack of restoring field in the second. 


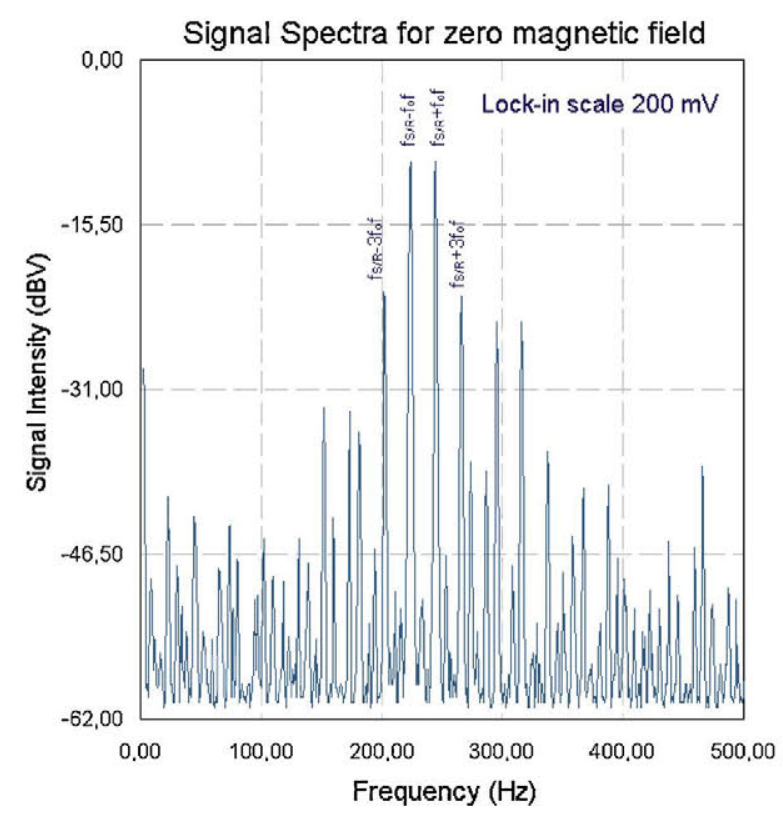

Fig. 13. Single-sided spectrum of the ideal AMR (upper graph) and the noisy AMR (lower graph) straps in the absence of an applied field.

The method consists in the following. Consider that the sensor is run in set-reset mode being the excitation frequency of this carrier signal $\omega$, and the offset coils are used to apply an alternating saturating field at a frequency $\left(\omega_{p}\right)$ one-to-two orders of magnitude lower than $\omega$. The output signal delivered to the lock-in amplifier can be expressed as:

$$
\begin{aligned}
x(t)= & \frac{\operatorname{sgn}\left\lfloor\sin \left(\omega_{p} t\right)+1\right\rfloor}{2}[r(t)-s(t)] \\
& +\frac{\operatorname{sgn}\left\lfloor\sin \left(\omega_{p} t\right)-1\right\rfloor}{2}[r(t)-s(t)]
\end{aligned}
$$

where $r(t)$ and $s(t)$ are the measurements in set-reset previously defined, and $\operatorname{sgn}(x)$ is the signum function defined as:

$\operatorname{sgn}(x)=\left\{\begin{array}{l}1, x \geq 0 \\ 0, x<0\end{array}\right.$

The signal of Eq. (13) is amplitude modulated. In the absence of an external dc field has a 50\% duty cycle. The Fourier transform of this signal for a HMC1002 AMR resistor [18] consists of a set of harmonics at the frequencies: $\omega \pm(2 n+1) \omega_{\mathrm{p}}$ with $n=0,1,2$, 3... (Fig. 13). In this example $\omega=2 \pi f_{S / R} \mathrm{rad} / \mathrm{s}$ with $f_{S / R}=224 \mathrm{~Hz}$, and $\omega_{\mathrm{p}}=2 \pi f_{\text {of }} \mathrm{rad} / \mathrm{s}$ with $f f_{\text {of }}=10 \mathrm{~Hz}$.

In the presence of a dc external magnetic field, the duty cycle of the modulation changes. This shift can be observed in Fig. 14 for real set up with an applied magnetic field of $50 \mu \mathrm{T}$.

This asymmetry in the signal leads to the appearance of new peaks at frequencies $\omega \pm 2 m \omega_{p}, m=0,1,2,3 \ldots$ (Fig. 15).

The peaks with frequencies $\omega \pm(2 n+1) \omega_{p}$ with $n=0,1,2,3 \ldots$ corresponds to the measurement in amplitude (common mode) similar to that of the set-reset method explained before. Among the peaks with frequencies $\omega \pm 2 m \omega_{p}$ with $m=0,1,2,3 \ldots$ the peak with $m=0$ is thought to correspond to the dc contribution to the signal and affected by a high noise $(1 / f)$ and the peaks with $m \geq 1$ are related to the time asymmetry.

The field can be measured with a double demodulation or with a lock-in at the $\omega \pm 2 \omega_{p}$ frequencies. These two methods are completely equivalent, so in this work we focus on the second one. To do a lock-in at the $\omega \pm 2 \omega_{p}$ frequencies and to avoid synchronism

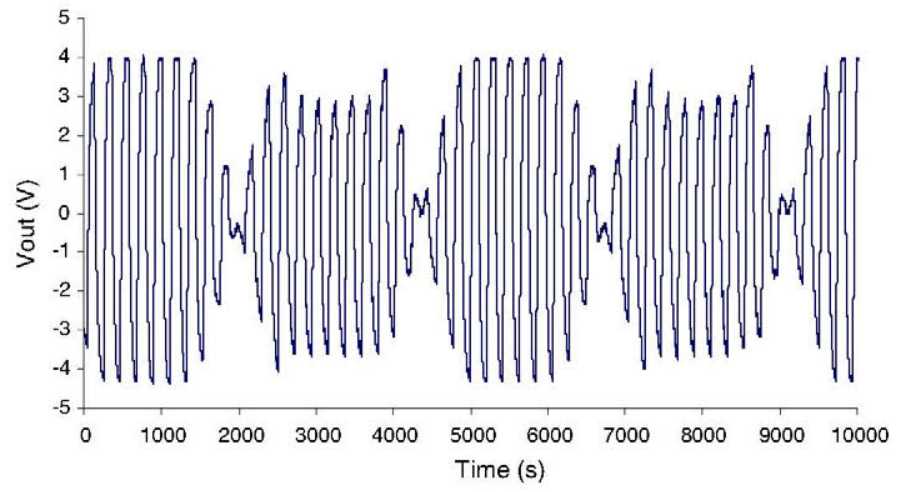

Fig. 14. Experimental output signal of the set up in the presence of a field of $50 \mu \mathrm{T}$ showing the effect on the modulation.

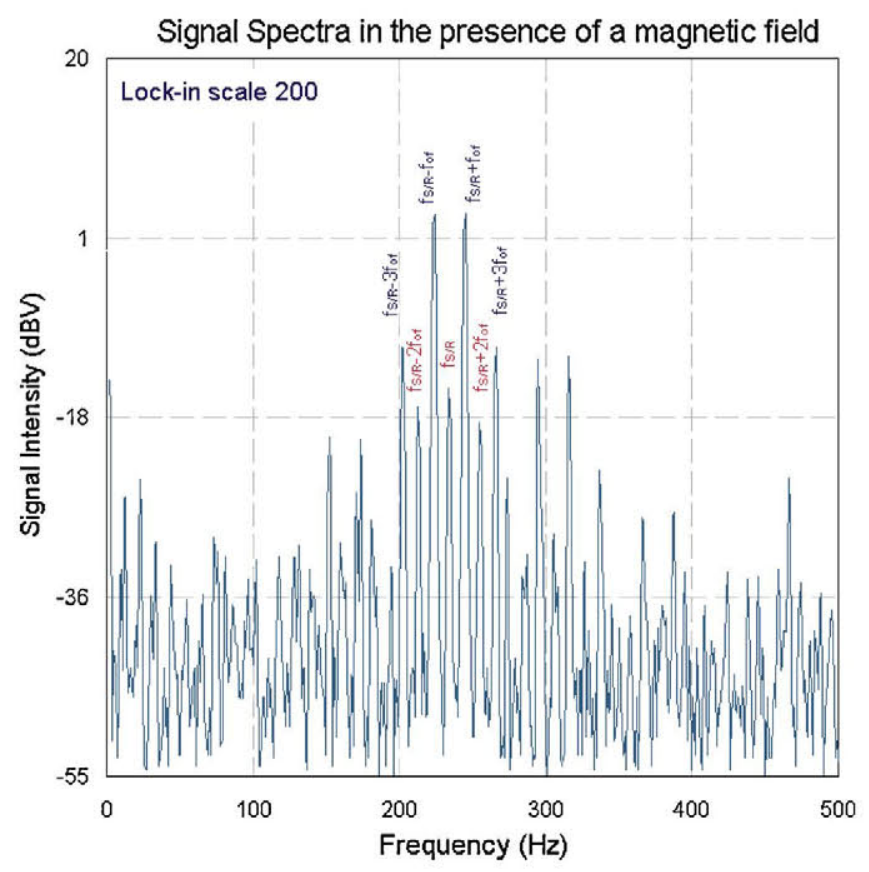

Fig. 15. Single-sided spectrum of the AMR device in the presence of a $50 \mu \mathrm{T}$ applied field.

problems all the frequencies have been generated with the same clock, and the frequencies have been calculated by means of a system of dipophantine equations subdividing conveniently the period of the signals as it has been explained in the methodology.

Even though the optimum measurements are considered to be in the $\omega \pm 2 \omega_{p}$ peaks, experimentally, these peaks are highly affected by the common mode, which makes it difficult to extract the field by the measurement of the time shift.

Because of this, all the arguments of the integrals for the different harmonics have been calculated to study if there is an algebraic combination to reduce the common mode. The result of the study was not successful. To be able to reach the limits in sensitivity, a series-opposition AMR circuit will have to be included to get rid of the common mode.

The response of the curves as a function of the field and the resolution (minimum detectable field) have been characterized for the $\omega \pm 2 \omega_{p}$ peaks. The sensors present a linear response as a function 


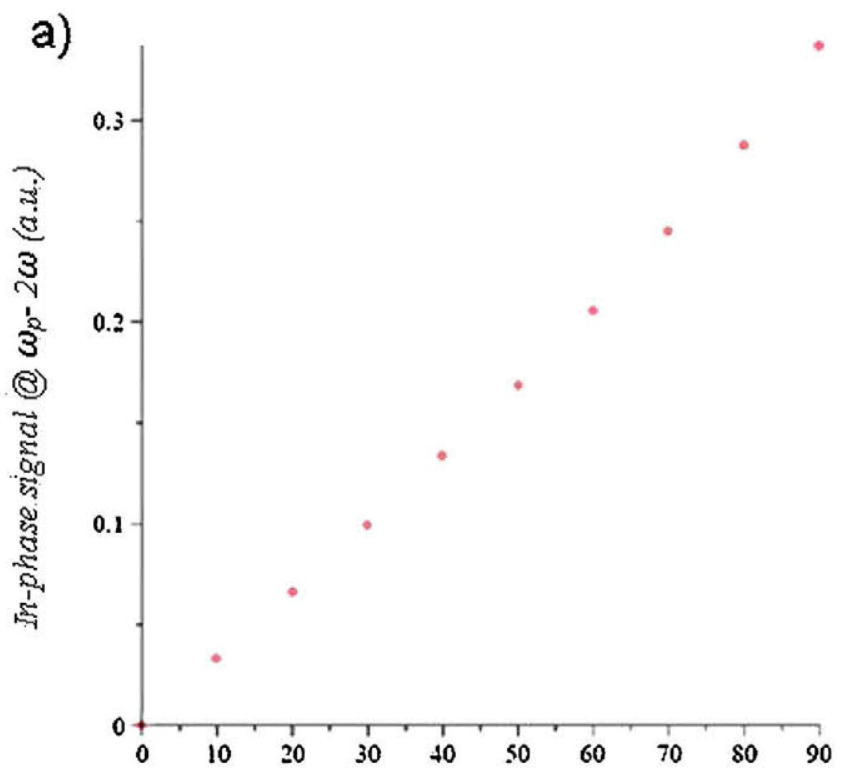

b)
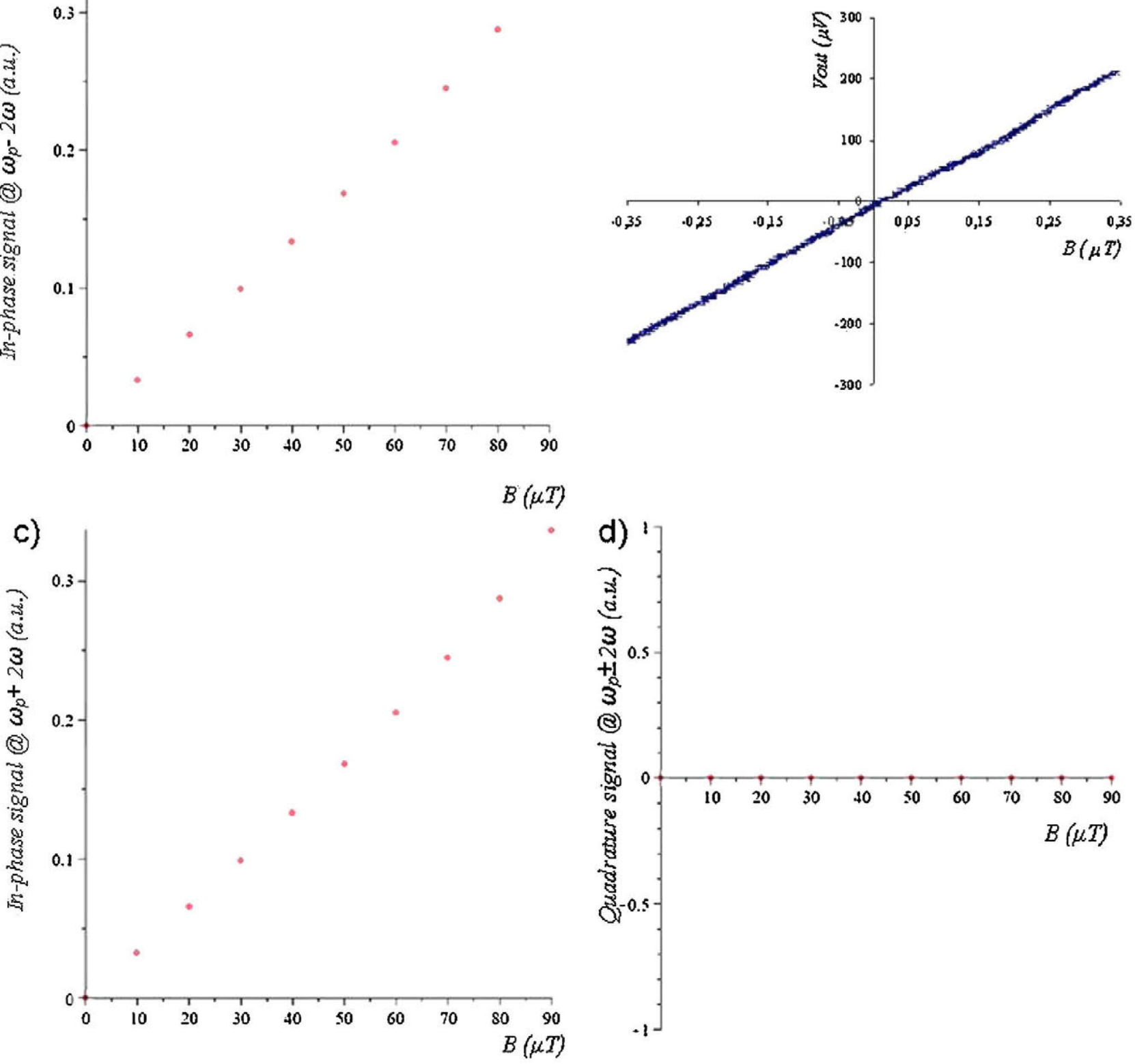

Fig. 16. In-phase and quadrature $\omega \pm 2 \omega_{p}$ harmonics: (a) In-phase $\omega-2 \omega_{p}$; (b) Experimental measurement at $\omega-2 \omega_{p}$; (c) In-phase $\omega+2 \omega_{p}$; (d) Quadrature $\omega \pm 2 \omega_{p}$.

of the field for the $\omega \pm 2 \omega_{p}$ harmonics as it was expected by the numerical integrations:

$$
\begin{aligned}
x\left(\omega_{p}-2 \omega\right)_{\text {in-phase }} & =\int_{-\pi / 2 \omega}^{\pi / 2 \omega} x(t) \sin \left(\omega_{p}-2 \omega\right) t \mathrm{~d} t \\
x\left(\omega_{p}-2 \omega\right)_{\text {quadrature }} & =\int_{-\pi / 2 \omega}^{\pi / 2 \omega} x(t) \cos \left(\omega_{p}-2 \omega\right) t \mathrm{~d} t \\
x\left(\omega_{p}+2 \omega\right)_{\text {in-phase }} & =\int_{-\pi / 2 \omega}^{\pi / 2 \omega} x(t) \sin \left(\omega_{p}+2 \omega\right) t \mathrm{~d} t \\
x\left(\omega_{p}+2 \omega\right)_{\text {quadrature }} & =\int_{-\pi / 2 \omega} x(t) \cos \left(\omega_{p}+2 \omega\right) t \mathrm{~d} t
\end{aligned}
$$

Fig. 16 shows the result of the numerical integration of these equations as a function of the field (a: in-phase signal at $\omega-2 \omega_{p}$, c: in-phase signal at $\omega+2 \omega_{p}$, d: quadrature signal at $\omega-2 \omega_{p}$, ) and a real measurement in our experimental set up (b). It can be seen that the in-phase signal locked at both frequencies is linear with the applied field.

Fig. 17 shows the Fourier transform for a $6.4 \mathrm{nT}$ amplitude external applied field of a $30 \mathrm{mHz}$ frequency. The green plot corresponds with the (squared) external field and the blue plot with the AMR response. Compared to method one, the relative amplitude of the peak in the Fourier transform is lower because the energy of the signal at this frequency is much lower. However, this method combined with the temperature correction described in the previous subsection (with the frequency $\omega+\omega_{p}$ as first harmonic equivalent), is proposed to provide repeatable, low noise and temperature immune measurements with AMR sensors. 


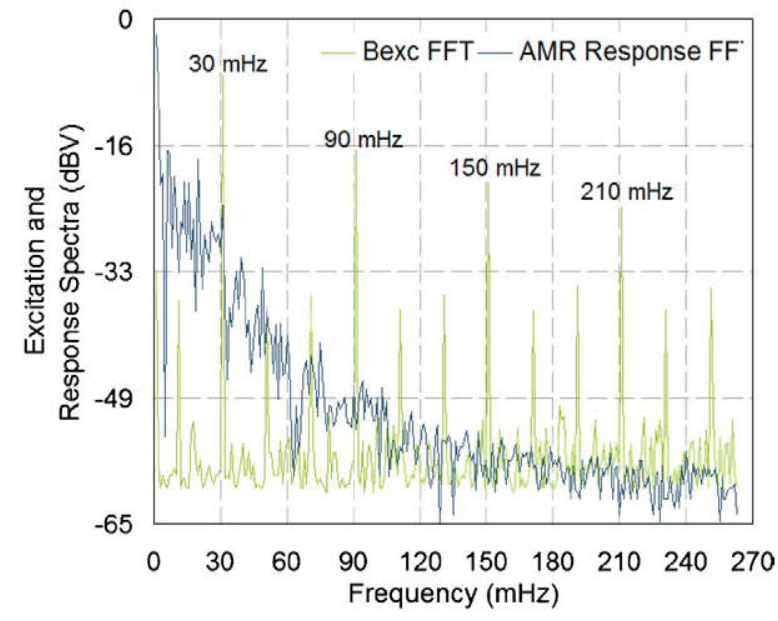

Fig. 17. Fouriertransform of the excitation signal: $6.4 \mathrm{nT}$ amplitude, $30 \mathrm{mHz}$ squared signal (green) and the in-phase output AMR at $\omega+2 \omega_{p}$ (blue). (For interpretation of the references to color in this figure legend, the reader is referred to the web version of this article.)

\section{Discussion}

The three methods described below help to improve the signalto-noise ratio (SNR) in AMR sensors since all of them provide a way to increase the frequency of the acquisition and the in-phase detection helps to filter the rest of the spectrum noise contribution. Therefore, the three methods permit a better retrieval of the external magnetic field reaching minimum detectable fields in the subnanoTesla range.

It can be said that the second method of measurement potentially improves the resolution respect to the first one since on the first hand, it consists in a time based measurement instead of an amplitude based one. And on the other hand, it offers the possibility to fully compensate the temperature measuring second and first harmonics due to the fact that first and second harmonics are dependent on temperature but only the second harmonic is affected by the field. The only open question of this method is the restoring mechanism needed in AMR sensors because of its comparative low anisotropy (of shape).

In the present work, we suggest the use of the third method proposed to achieve a mechanism of measurement with AMR capable to perform the restoring to zero, which is temperature compensated and robust against noise. In a future work a full characterization of the proposed set up will be presented.

\section{Conclusions and further work}

Three different methods for AMR operation in frequency have been described and explained:

1 A lock-in amplifier with the set-reset excitation.

2 Fluxgate like operation of the AMR provided with temperature correction.

3 A mixed method with double demodulation or direct lock-in at the sum and difference frequencies of the saturation and the flipping frequencies.

The first method is an amplitude measurement, the second a time measurement, which allows to correct the temperature dependence of AMR sensors and the third one a combination of both, which guarantees the repeatability avoiding the sensitivity loss with the constant flipping of the spins in the magnetic stripes.

We propose the use of this third method for applications when AMR sensors are suitable regarding the magnetic requirements but the temperature swing is high because it implies a magnetic field measurement more robust against noise and temperature independent.

Next step is to build up a set to fully characterize this third method with field, noise and temperature.

The set up will consist of a couple of AMR sensors in series opposition to be able to measure the first (at $\omega+\omega_{p}$ ) and second harmonics (at $\omega+2 \omega_{p}$ ). The electronics will be done with a field programmable gate array (FPGA) in which the frequencies will be generated by the same clock to avoid problems of synchronization. The signals will be generated by means of a couple of digital lock-ins at $\omega$ and $\omega+2 \omega_{p}$ programmed in the FPGA.

Finally we will implement this design in an ASIC for maximum compactness and miniaturization.

\section{Acknowledgments}

This work has been partially supported by the MICINN grant AYA2011 -29967-C05 -01 for Meiga-MetNet Project and MAT 2011 28751-C02-01 grant. The authors are also in debt with M. Perez and with SIMAVE SA for the use of the magnetic sensors

\section{References}

[1] M. D.Michelena, I, Arruego, J.M. Oter, H. Guerrero, CoTS-based wireless magnetic sensor for small satellites, IEEE Trans. Aerosp. Electron. Syst. 46 (2) (2010) $542-557$.

[2] M. Díaz-Michelena, Small magnetic sensors for space applications, Sensors 9 (2009) 2271-2288.

[3] N. Smith, F. Jeffers, J. Freeman, A high sensitivity magnetoresistive magnetome ter, J. Appl. Phys. 69 (8) (1991) 5082-5084.

[4] A.S. Edelstein, Advances in magnetometry, J. Phys.: Condens. Matter 19 (2007) 165217.

[5] J. Vyhnanek, et al., Low frequency noise of anisotropic magnetoresistors in DC and AC excited metal detectors, J. Phys.: Conf. Ser. 450 (2013) 012031.

[6] E. Zimmermann, A. Verweerd, W. Glaas, A. Tillmann, A. Kemna, An AMR sensorbased measurement system for magnetoelectrical resistivity tomography, IEEE Sens. J. 5 (2005) 233-241.

[7] P. Ripka, M. Janošek, M. Butta, W.S. Billingsley, E.M. Wakefield, Crossedfield effect in commercial fluxgate and AME sensors, J. Electr. Eng. 61 (2010) $13-16$.

[8] Brown P., Whiteside B. J., Beek T. J., Fox P., Horbury T. S., Oddy T. M., Archer M. O., Eastwood J. P., Sanz-Hernández D., Sample J. G., Cupido E., O’Brien H. and Carr C. M., Space magnetometer based on an anisotropic magnetoresistive hybrid sensor, Rev. Sci. Instrum. (in press).

[9] P.A.M. Sandborn, P.A. Sandborn, Using embedded resistor emulation and trimming to demonstrate measurement methods and associate engineering model development, Int. J. Eng. Ed. 22 (2006) 1-7.

[10] P. Ripka, M. Butta, A. Platil, Temperature stability of AMR sensors, Sens, Lett. 11 (2013) 74-77.

[11] M.Vopalensky, A. Platil, Temperature drift of offset and sensitivity in full-bridge magnetoresistive sensors, IEEE Trans. Magn. 49 (1) (2013) 139.

[12] N.A.Stutzke, S.E. Russek, D.P. Pappas, M.Tondra, Low-frequency noise measurements on commercial magnetoresistive magnetic field sensors, J. Appl. Phys. 97 (2005) $10 Q 107$.

[13] N.A. Stutzke, S.E. Russek, D.P. Pappas, Low-frequency noise measurements on commercial magnetoresistive magnetic field sensors, J. Appl. Phys. 97 (2005) $10 Q 107$.

[14] Mario H. Acuña, Space-based magnetometers, Rev. Sci. Instrum. 73 (11)(2002) $3717-3736$

[15] F. Primdahl, The fluxgate magnetometer, J. Phys., E: Sci. Instrum. 12 (1979) 241.

[16] P. Brown, T. Beek, C. Carr, H. O'Brien, E. Cupido, T. Oddy, T.S. Horbury, Corrigen dum: magnetoresistive magnetometer for space science applications, Meas. Sci. Technol. 23 (2012) 059501.

[17] P.D. Dimitropoulos, J.N. Avaritsiotis, Integrating the fluxgate principle in the spin-valve and AMR sensor technologies, Sens. Actuators, A: Phys. 106 (1) (2003) 43-47.

[18] Honeywell datasheet HMC1002. www.honeywell.com. 


\section{Biographies}

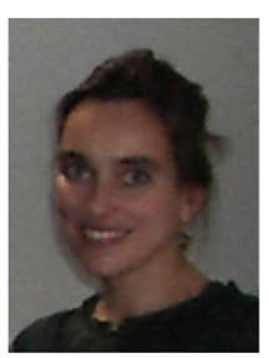

Marina Díaz-Michelena Ph.D in Physics from Polytechnic University of Madrid (2004). Current position: head of the Space Magnetism Laboratory at the Spanish National Institute or Aerospace Technology (INTA). Dr. Díaz Miche lena has evolved a line in miniaturized and compact magnetometers for space and planetary applications. The magnetometers in Earth orbit are currently being used for Space weather purposes. As a result of this development activity she has grown an interest in planetary magnetometry. In particular devoted to develop new instruments for Mars and Moon magnetic mineralogy as well as in the investigation of terrestrial analogs.

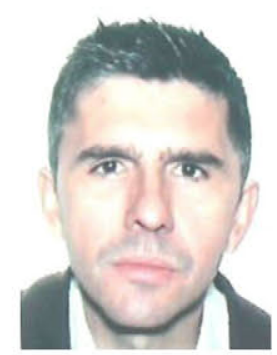

Pedro Cobos Arribas Ph.D in Telecommunication Engineering from Polytechnic University of Madrid (2001). Current position: Titular professor on the ETSIST, UPM Madrid. Researcher at the Institute of Optoelectronic Systems and Microtechnology (ISOM).

Dr. Cobos Arribas did his first research work with a fluxgate application, as his Final year Proyect, in 1990. $\mathrm{He}$ is currently involved in the design and development of microcontroller circuits solutions for magnetic applications, as linear motor controllers, magnetic detectors and magnetic detection instruments.

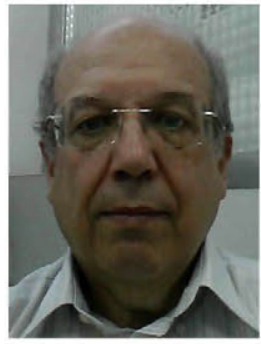

Claudio Aroca Hernández-Roz Ph.D in Physics from the Complutense University of Madrid (1980). Current position: Prof. Dr. Head of the Department of Physics Applied to the Information Technologies Department of the Polytechnic University of Madrid. Subdirector of the Institute of Optoelectronic Systems and Microtechnology (ISOM).

Prof. Dr. Aroca started his research in the study of magnetic structures in amorphous magnetic materials. This research derived in an applied activity in the field of magnetic sensors and instrumentation. At ISOM he has focused on thin film materials study, the exchange interac tion in these systems, and development of planar sensors. He is currently involved in the design and development of spintronic based devices and in the study of magnetic nanoparticles and its application for biomedical technologies as NMR contrast and MEG. 